\title{
Reduction of HIV transmission rates from mother to child in the era of antiretroviral therapy in the Lake Victoria zone, Tanzania
}

\author{
MARIAM M. MIRAMBO ${ }^{*}$, CELINE SIMON ${ }^{1}$, ALPHAXARD KAJURA ${ }^{1}$, BENSON KIDENYA ${ }^{1}$, MARTHA F.MUSHI', \\ MAJIGO MTEBE ${ }^{2}$ and STEPHEN E. MSHANA ${ }^{1}$ \\ ${ }^{1}$ Weill Bugando School of Medicine, Catholic University of Health and Allied Sciences-Bugando, Mwanza, \\ Tanzania \\ ${ }^{2}$ Muhimbili University of Health and Allied Sciences, Dar es Salaam, Tanzania
}

\begin{abstract}
Background: Since the introduction of prevention of mother to child transmission (PMTCT) in Tanzania, HIV infection rates have been reduced in different regions across the country. However, there is limited published data from the Lake Victoria zone of Tanzania regarding the effectiveness of various regimens used for PMTCT. This study was done to assess the effectiveness of antiretroviral therapy in preventing mother to child transmission of HIV

Methods: Infants aged $\leq 18$ months born to HIV positive mothers undertaking PMTCT programme and those with no intervention program from Mara, Kagera, Mwanza and Shinyanga were tested for HIV-1 DNA polymerase chain reaction (PCR). Data were analysed using STATA version 10.0 to assess factors associated with outcome.

Results: A total of 1,005 study subjects were enrolled in the study. Of these $55 \%$ (554/1005) were females. Majority (82.6\%; 830/1005) of the infants studied were aged 1-6 months. The median age of the infant studied was 3 months (IQR 2-4). Out of 1005 non-repetitive samples; 61(6.1\%) were HIV-1 DNA PCR positive. Positive dried blood spots (DBS) rates by region were $6.4 \%, 5.9 \%, 5.6 \%$ and $5.1 \%$ in Mwanza, Mara, Kagera and Shinyanga, respectively. During pregnancy interventions, the positive rate for women with no therapy was $12.6 \%$ and for zidovudine alone was $5.4 \%$ while for triple antiretroviral therapy was $0.5 \%$. Women who were in highly active antiretroviral therapy (HAART) during pregnancy had significantly lower positive rate than those without HAART treatment $(p=0.001)$. Of 755 infants who received nevirapine, $3.9 \%$ were DBS positive compared to $12.8 \%$ of those who didn't receive nevirapine ( $p=0.001)$.

Conclusion: The use of antiretroviral therapy in the PMTCT programme is effective in reducing HIV transmission from mother to child.
\end{abstract}

Keywords: HIV, ART, PMTCT, mother, infant, transmission, Tanzania

\section{Introduction}

Human immunodeficiency virus (HIV) transmission from mother to child may occur during pregnancy, delivery or through breastfeeding. It has been observed that about $25 \%-45 \%$ of children may become infected in the absence of prevention intervention (Cock et al., 2000). Previous reports indicate that about one third of transmissions occurs through breastfeeding (John-Stewart et al., 2004), while $70 \%$ of the remaining occur during delivery and $30 \%$ during pregnancy (Cock et al., 2000). Infants who acquire HIV during pregnancy and around the time of delivery progress rapidly to AIDS or death in the first few months of life (Ciaranello et al., 2011). In many resource rich countries mother to child HIV transmission has been reported to be below $2 \%$. This low level has been achieved through different approaches including provision of different combinations of highly active antiretroviral therapies (HAART), formula feeding of infants and elective caesarean sections (Giaquinto et al., 2006).

The situation is different from resource limited countries whereby in 2013 more than 150,000 new infections were observed among children (UNAIDS, 2012). Implementation of prevention of mother to child transmission (PMTCT) services including screening pregnant women and provision of antiretroviral therapies in many resource limited countries is important to achieve the goal of reducing HIV infection globally. In Tanzania, about one-fifth of all HIV new

\footnotetext{
${ }^{*}$ Correspondence E-mail: mmmirambo@gmail.com
} 
infections are due to mother-to-child transmission. To avoid mother-to-child transmission, antiretroviral treatment for the mother and baby has been integrated into routine reproductive health services. Tanzania's PMTCT programme was introduced in 2000 and by 2011 about threequarters of all HIV positive pregnant women in need of ARV therapy had received it (MoHSW, 2007; UNAIDS, 2012).

PMTCT interventions provide a critical opportunity to prevent vertical transmission of HIV from mother to child during pregnancy, labour and delivery. The most important part in measuring its effectiveness is by assessing the infection rate in new-borns born to HIV positive mothers who received PMTCT services. Different studies (Rutta et al., 2008; Gamell et al., 2013) have been done to assess HIV infection rates but there is limited data in the Lake Victoria zone. Therefore this study was done to assess the effectiveness of ART in reducing transmission rates of HIV from mother to child in the Mwanza, Mara, Shinyanga and Kagera Regions of, Tanzania.

\section{Materials and Methods}

\section{Study area and population}

This cross sectional study was carried out in the lake Victoria Zone of Tanzania from June to July 2013, covering regions of Kagera, Mwanza, Shinyanga and Mara. The study included a total of 1,005 dry blood spots (DBS) of infants aged $\leq 18$ months born to HIV seropositive mothers. Of 1,005 infant's DBS, 766 were from women undertaking PMTCT programme of which 209 were on HAART and 557 were on zidovudine (AZT). Among women on HAART in some regions they had started combination of tenofovir (TDF) +lamivudine (3TC) and efavirenz (EFV) while in other regions they were still using $A Z T+3 T C+N V P$. Two hundred and thirty nine DBS samples were from infants whose mothers who were not on any therapy. All DBS of which dates of collection were absent and those with the same birth date as sample collection date were excluded. Maternal and infant regime data were obtained from PMTCT follow up register.

\section{Sample collection and processing}

All DBS samples were transported to Bugando Medical Centre (BMC) reference laboratory in Mwanza for processing. All samples with valid results were included in the analysis. Sample size was calculated by using Kish Lisle formula. In all health facilities DBS were prepared on Whatman filter paper (Sigma-Aldrich, Nairobi Kenya) using heel stick blood sample obtained from infants who were born from HIV-1 positive mothers. The samples were dried and stored at room temperature, and then transported to BMC laboratory in a plastic resalable bag which contains absorbents and humidity meter. The AMPLICOR HIV-1 DNA Test, version 1.5 (Roche Products (Pty) Ltd - South Africa) was used to detect HIV DNA in DBS samples as previously described(Nsojo et al., 2010).

\section{Data analysis}

Analysis was carried out using STATA version 10.0. Chi square was used to evaluate association of data on $2 \times 2$ contingency tables, effectiveness of PMTCT intervention service was calculated using $Z$ test. A p-value $<0.05$ was considered statistically significant.

\section{Ethical considerations}

Ethical clearance was sought from the Joint CUHAS/ BMC Research Committee (CREC). Using documented sources of samples all results from this study were sent back to the patients for appropriate patient's management. 


\section{Results}

Social demographic characteristics of the study population

Of the 1,005 studied samples $55 \%$ (554/1005) were from female subjects. Majority $82.6 \%(830 / 1005)$ of the infants were aged 1-6 months. The median age of the infants was 3 months (Interquartile range $=2-4$ ) with even distribution between infants from mothers with intervention and those without intervention (Table1).

Table 1: Socio-demographic characteristic of the infants

\begin{tabular}{lll}
\hline Variables & Frequency & Percent \\
\hline Sex & & \\
Female & 554 & 55.1 \\
$\quad$ Male & 451 & 44.9 \\
Age(months) & & \\
$1-6$ & 830 & 82.6 \\
$7-12$ & 151 & 15 \\
$13-18$ & 24 & 2.4 \\
Residence & & \\
Mwanza & 249 & 24.7 \\
Mara & 251 & 25 \\
Kagera & 252 & 25.1 \\
Shinyanga & 253 & 25.2 \\
\hline
\end{tabular}

\section{HIV-1DNA PCR results}

Of the 1,005 samples, 61 (6.1\%) were HIV-1 DNA PCR positive. The prevalence of HIV-1 was highest among infants from Mwanza. However, the regional difference between prevalence was not statistically significant (Table 2). The prevalence was significantly higher in male infants than females $(p=0.011)$; it was also significantly lower among those from mothers under the intervention $(p=0.001)$.

Table 2: HIV- 1 DNA PCR results, Socio demographic factors and PMTCT intervention

\begin{tabular}{lllll}
\hline Infant characteristic & $\begin{array}{l}\text { Positive } \\
(\mathbf{n} \%)\end{array}$ & $\begin{array}{l}\text { Negative } \\
(\mathbf{n} \%)\end{array}$ & Total & P value \\
\hline Residence & $16(6.4)$ & $233(93.6)$ & 249 & 0.935 \\
Mwanza & $15(5.9)$ & $236(94.1)$ & 251 & \\
Mara & $14(5.6)$ & $238(94.4)$ & 252 & \\
Kagera & $13(5.1)$ & $240(94.9)$ & 253 & \\
Shinyanga & & & & \\
Age(months) & $50(6)$ & $780(94)$ & 830 & 0.776 \\
$1-6$ & $9(6)$ & $142(94)$ & 151 & \\
$7-12)$ & $2(8.3)$ & $22(91.7)$ & 24 & \\
$13-18)$ & & & & \\
Sex & $24(4.3)$ & $530(95.7)$ & 554 & 0.011 \\
Female & $37(8.2)$ & $414(91.8)$ & 451 & \\
Male & & & & \\
PMTCT intervention & $41(4.5)$ & $867(95.5)$ & 908 & 0.001 \\
Yes & $20(20.6)$ & $77(79.4)$ & 97 & \\
$\quad$ No & & & & \\
\hline
\end{tabular}


Of 209 women who were on HAART during pregnancy and delivery one (0.5\%) infant DBS was positive for HIV- 1 DNA. Out of 239 women who did not receive any ARV therapy during pregnancy and delivery, $30(12.6 \%)$ had positive HIV- 1 DNA PCR. Of the women who received AZT during pregnancy, 5/4\% (30/557) had positive HIV-1 DNA PCR compared to 12.6\% (30/239) from mothers with no therapy (Table 3 ). The use of antiretroviral drugs during delivery was associated with significant decrease DBS positive rates $(p=0.001)$.

Table 3: Drug regimen of (mother and infant) and HIV- 1 DNA PCR result of infants

\begin{tabular}{|c|c|c|c|c|}
\hline \multirow[b]{2}{*}{ Drug regimen } & \multicolumn{3}{|c|}{ HIV-1 DNA PCR results of infant } & \multirow[b]{2}{*}{ p-value } \\
\hline & $\begin{array}{l}\text { Positive } \\
\text { n (\%) }\end{array}$ & $\begin{array}{c}\text { Negative } \\
\text { n (\%) }\end{array}$ & Total & \\
\hline \multicolumn{5}{|l|}{ Mother in pregnancy } \\
\hline $\mathrm{TDF}+3 \mathrm{TC}+\mathrm{EFV}$ & $1(0.5)$ & $208(99.5)$ & 209 & 0.001 \\
\hline AZT & $30(5.4)$ & $527(94.6)$ & 557 & \\
\hline No therapy & $30(12.6)$ & $209(87.5)$ & 239 & \\
\hline \multicolumn{5}{|c|}{ Mother during delivery } \\
\hline $\mathrm{TDF}+3 \mathrm{TC}+\mathrm{EFV}$ & $1(0.5)$ & $208(99.5)$ & 209 & 0.001 \\
\hline sdNVP & $4(2.5)$ & $159(97.5)$ & 163 & \\
\hline $\mathrm{NVP}+\mathrm{AZT}+3 \mathrm{TC}$ & $4(1.4)$ & $278(98.6)$ & 282 & \\
\hline No therapy & $30(12.6)$ & $209(87.4)$ & 239 & \\
\hline Missed information & $22(19.6)$ & $90(80.4)$ & 112 & \\
\hline \multicolumn{5}{|c|}{ Infant therapy after birth } \\
\hline NVP & $29(3.9)$ & $726(96.1)$ & 755 & 0.001 \\
\hline No therapy & $32(12.8)$ & $218(87.2)$ & 250 & \\
\hline
\end{tabular}

\section{Discussion}

Results from this study suggest that ART are effective in reducing mother-to-child transmission of HIV in the target population. Lake Victoria Zone had a prevalence of $6.1 \%$ regardless of PMTCT intervention utilization. This indicates declining rate as compared to findings of the previous study (Nuwagaba-Biribonwoha et al., 2010) which reported a 17\% transmission rate regardless of PMTCT intervention. The slightly higher HIV prevalence in Mwanza could be explained by the more access to health facilities in the city which facilitate increase of HIV testing and identification of positive cases.

In our study setting PMTCT intervention was associated with significant decrease in transmission of HIV. Similar findings have been previously in three regions in northern Tanzania (Buchanan et al., 2014). However, its practice is facing challenges in infants diagnosis (Chiduo et al., 2013). Our findings are comparable to a study in Zambia which observed lower transmission rates among infants/mother who received antiretroviral therapy than those who did not receive (Stringer et al., 2005). In addition our study confirmed earlier observations in northern Tanzania regarding HIV transmission rate (Buchanan et al., 2014).

Based on drug regimen, women who did not get any therapy during pregnancy had higher transmission rates than those who used AZT or those on HAART. These findings support the use of HAART during pregnant in reducing transmission rate. Prevalence of HIV is lower in infants whose mothers received HAART than those who received AZT alone. Similarly, single dose nevirapine (sdNVP) 6 months after birth is effective in reducing transmission rate (Coovadia et al., 2012). Moreover, in the present study single dose AZT was effective in reducing MTCT (Connor et al., 1994).

Women who used alternative first line regimen $(\mathrm{NVP}+\mathrm{AZT}+3 \mathrm{TC})$ had a lower transmission rate than that documented by WHO (2007). However, there is missing information in some 
women regarding the therapy used during delivery and some did not get therapy during delivery. This could partly be due to the poor adherence "take-home" of ARV for mother and newborn when given to HIV positive women at antenatal clinic and insufficient use of facility-based delivery. Similar observations have been reported in previous studies (Cook et al., 2011; Musoke et al., 1999). Infants who were not given NVP after birth had high transmission rate than to those who were given NVP. It was not clearly documented how long the infants used NVP. However, following the national PMTCT guidelines (Gamell et al., 2013) the dose should be up to six weeks of age. Provision of further therapy is recommended especially in low income countries to sustain the benefit of PMTCT through breastfeeding (Team, 2002).

In the present study, administration of sdNVP only during delivery reduced the transmission rate to $2.5 \%$ which is lower than rates reported by others (Lallemant et al., 2004; Taha et al., 2004). This suggests that sdNVP is still a cost effective intervention compared to triple therapy. It has potent antiviral activity and is rapidly absorbed and passed through the placenta (Minkoff \& Augenbraun, 1997). As most HIV transmission occurs during active labour, rapid intrapartum interventions can impact transmission significantly. NVP has been shown to decrease HIV-RNA viral load by 1.3 logs after a single dose at 1 week postpartum (Musoke et al., 1999).

The use of HAART in this study have been shown to be more effective in reducing MTCT compared to single therapy and similarly to those without any therapy. Our results are comparable to findings of a previous study by Chasela et al., (2010) and consistent with the recent WHO recommendations (WHO, 2009). This also has been observed in previous intervention programme in the Haydom Lutheran Hospital in northern Tanzania (Evjen-Olsen et al., 2009; Johannessen et al., 2009). Our study reported a lower rate as compared to the rate among pregnant women on HAART elsewhere (Cooper et al., 2002; Stinson et al., 2010). Despite the effectiveness of this approach, it is associated with high cost that might hinder the goal of reduction of MTCT especially in low income countries like Tanzania (Robberstad \& Evjen-Olsen, 2010).

In conclusion, the findings from this study suggest that PMTCT programme in the Lake Victoria zone of Tanzania is effective in reducing the prevalence of HIV infection. The use of HAART is therefore important in reducing MCTC and this can be achieved by close monitoring of maternal health through increasing HIV testing among pregnant women and identify those in need of treatment.

\section{Competing interest}

The authors declare that they have no competing interests

\section{Author's contribution}

AK, MMM, BK, participated in designing the study, CS did data collection, BK did data analysis. MMM, MFM, MM and SEM participated in results interpretation and preparation of the manuscript. MMM and SEM wrote the manuscript which was revised and approved by all authors.

\section{Acknowledgements}

We are very grateful to the care and treatment centre, Bugando Medical Centre (BMC). We would like to acknowledge the assistance and guidance provided by all staff members of the BMC laboratory, as well as the Department of Microbiology/Immunology, Catholic University of Health and Allied Sciences and BMC. This research was supported by grants from Ministry of Health and Social Welfare. 


\section{References}

Buchanan, A. M., Dow, D. E., Massambu, C. G., Nyombi, B., Shayo, A., Musoke, R., Feng, S., Bartlett, J. A., Cunningham, C. K. and Schimana, W. (2014) 'Progress in the Prevention of Mother to Child Transmission of HIV in Three Regions of Tanzania: A Retrospective Analysis', PloS one 9(2): e88679.

Chiduo, M.G., Mmbando, B.P., Theilgaard, Z.P., Bygbjerg, I.C., Gerstoft, J., Lemnge, M. \& Katzenstein, T.L. (2013) Early infant diagnosis of HIV in three regions in Tanzania; successes and challenges. BMC Public Health 13(1): 910.

Ciaranello, A.L., Park, J.E., Ramirez-Avila, L., Freedberg, K.A., Walensky, R.P. \& Leroy, V. (2011) Early infant HIV-1 diagnosis programs in resource-limited settings: opportunities for improved outcomes and more cost-effective interventions. BMC Medicine 9(1): 59.

Cock, K.D., Fowler, M., Mercier, E., Vincenzi, I.D., Saba, J., Hoff, E., Alnwick, D., Rogers, M. \& Shaffer, N. (2000) Special communication-prevention of mother-to-child HIV transmission in resource-poor countries: translating research into policy and practice. Journal of the American Medical Association 283, 1175-1182.

Connor, E.M., Sperling, R.S., Gelber, R., Kiselev, P., Scott, G., O'Sullivan, M.J., Van Dyke, R., Bey, M., Shearer, W. \& Jacobson, R.L. (1994) Reduction of maternal-infant transmission of human immunodeficiency virus type 1 with zidovudine treatment. New England Journal of Medicine 331, 1173-1180.

Cook, R.E., Ciampa, P.J., Sidat, M., Blevins, M., Burlison, J., Davidson, M.A., Arroz, J.A., Vergara, A. E., Vermund, S.H. \& Moon, T.D. (2011) Predictors of successful early infant diagnosis of HIV in a rural district hospital in Zambezia, Mozambique. Journal of Acquired Immune Deficiency Syndromes 56(4): e104.

Cooper, E.R., Charurat, M., Mofenson, L., Hanson, I.C., Pitt, J., Diaz, C., Hayani, K., Handelsman, E., Smeriglio, V. \& Hoff, R. (2002) Combination antiretroviral strategies for the treatment of pregnant HIV-1-infected women and prevention of perinatal HIV-1 transmission. Journal of Acquired Immune Deficiency Syndromes 29(5), 484-494.

Evjen-Olsen, B., Olsen, Ø.E. \& Kvåle, G. (2009) Achieving progress in maternal and neonatal health through integrated and comprehensive healthcare services-experiences from a programme in northern Tanzania. International Journal for Equity in Health 8(1), 1-14.

Gamell, A., Letang, E., Jullu, B., Mwaigomole, G., Nyamtema, A., Hatz, C., Battegay, M. \& Tanner, M. (2013) Uptake of guidelines on prevention of mother-to-child transmission of HIV in rural Tanzania: time for change. Swiss Medical Weekly 143:w13775.

Giaquinto, C., Rampon, O. \& De Rossi, A. (2006) Antiretroviral therapy for prevention of motherto-child HIV transmission. Clinical Drug Investigation 26, 611-627.

Johannessen, A., Naman, E., Kivuyo, S.L., Kasubi, M.J., Holberg-Petersen, M., Matee, M.I., Gundersen, S.G. \& Bruun, J.N. (2009) Virological efficacy and emergence of drug resistance in adults on antiretroviral treatment in rural Tanzania. BMC Infectious Diseases 9(1): 108.

John-Stewart, G., Mbori-Ngacha, D., Ekpini, R., Janoff, E.N., Nkengasong, J., Read, J.S., Van de Perre, P. \& Newell, M.-L. (2004) Breast-feeding and transmission of HIV-1. Journal of Acquired Immune Deficiency Syndromes 35, 196.

Minkoff, H. \& Augenbraun, M. (1997) Antiretroviral therapy for pregnant women', American Journal of Obstetrics and Gynecology 176, 478-489.

MoHSH (2007) Health Sector HIV and AIDS Strategic Plan (HSHSP) 2008-2012. The Ministry of Health and Social Welfare-Tanzania Mainland.

Musoke, P., Guay, L.A., Bagenda, D., Mirochnick, M., Nakabiito, C., Fleming, T., Elliott, T., Horton, S., Dransfield, K. \& Pav, J.W. (1999) A phase I/II study of the safety and pharmacokinetics of nevirapine in HIV-1-infected pregnant Ugandan women and their neonates (HIVNET 006). AIDS 13, 479-486. 
Nsojo, A., Aboud, S. \& Lyamuya, E. (2010) Comparative evaluation of Amplicor HIV-1 DNA test, version 1.5 , by manual and automated DNA extraction methods using venous blood and dried blood spots for HIV-1 DNA PCR testing. Tanzania Journal of Health Research 12, 228232.

Nuwagaba-Biribonwoha, H., Werq-Semo, B., Abdallah, A., Cunningham, A., Gamaliel, J.G., Mtunga, S., Nankabirwa, V., Malisa, I., Gonzalez, L.F. \& Massambu, C. (2010) Introducing a multi-site program for early diagnosis of HIV infection among HIV-exposed infants in Tanzania. BMC Pediatrics 10(1): 44.

Robberstad, B. \& Evjen-Olsen, B. (2010) Preventing mother to child transmission of HIV with highly active antiretroviral treatment in Tanzania-a prospective cost-effectiveness study. Journal of Acquired Immune Deficiency Syndromes 55, 397-403.

Rutta, E., Gongo, R., Mwansasu, A., Mutasingwa, D., Rwegasira, V., Kishumbu, S., Tabayim J., Masini, T. \& Ramadhani, H. (2008) Prevention of mother-to-child transmission of HIV in a refugee camp setting in Tanzania. Global Public Health 3: 62-76.

Stinson, K., Boulle, A., Coetzee, D., Abrams, E.J. \& Myer, L. (2010) Initiation of highly active antiretroviral therapy among pregnant women in Cape Town, South Africa. Tropical Medicine \& International Health 15, 825-832.

Stringer, J.S., Sinkala, M., Maclean, C.C., Levy, J., Kankasa, C., DeGroot, A., Stringer, E.M., Acosta, E.P., Goldenberg, R.L. \& Vermund, S.H. (2005) Effectiveness of a city-wide program to prevent mother-to-child HIV transmission in Lusaka, Zambia. AIDS 19, 1309.

Team, P.S. (2002) Efficacy of three short-course regimens of zidovudine and lamivudine in preventing early and late transmission of HIV-1 from mother to child in Tanzania, South Africa, and Uganda (Petra study): a randomised, double-blind, placebo-controlled trial. Lancet 359 (9313), 1178.

UNAIDS (2012) World AIDS Day Report - Results' 55. 